\title{
Left atrial myxoma combined with coronary artery disease in an elderly patient
}

\author{
Gündüz Yümün, Selami Gürkan, Okan Donbaloğlu \\ Department of Cardiovascular Surgery, Namık Kemal University School of Medicine, Tekirdağ, Turkey
}

\begin{abstract}
Cardiac myxoma associated with coronary artery disease is a rare pathology in an elderly patient. Herein we present an 81-year-old woman undergoing simultaneous surgical treatment for left atrial myxoma combined with coronary artery disease. The postoperative course of the patient was uneventful. The initial removal of mass should be performed to prevent systemic embolization of tumor fragments.
\end{abstract}

Eur Res J 2018;4(2):129-131

Keywords: Left atrial mass, coronary artery disease, elderly

\section{Introduction}

Atrial myxomas are accounting for nearly half of primary cardiac tumors in adults [1]. Approximately $75-90 \%$ of cardiac myxomas are located in the left atrium, mainly adhered to the atrial septum near the fossa ovalis $[2,3]$. In most cases they encountered in female patients with 40-60 years old [1]. The size of the myxoma is directly proportional to the severity of the patient's symptoms [4]. Coexistence of coronary artery disease and myxoma is a rare condition and, it is reported in a limited number of case reports [5-8]. We describe a rare case with simultaneous left atrial myxoma and coronary artery disease in an elderly woman with shortness of breath and chest pain.

\section{Case Presentation}

An 81-year-old woman admitted to hospital with shortness of breath and chest pain. Upon physical examination, the patient had a heart rate of 78 beats/min, and a regular blood pressure of $170 / 70$ $\mathrm{mmHg}$. In the clinical history, she had undergone coronary angiography 3 years ago and coronary artery disease had been detected. There was no previous echocardiography report.

The systemic examination of the patient was normal. The electrocardiogram was also normal. Echocardiographic evaluation identified left ventricular apical akinesis with an ejection fraction of $45 \%$ and showed a left atrial mass $38 \times 32 \mathrm{~mm}$ in sizes attached to the interatrial septum of the left atrium without obstruction.

Coronary angiography showed plaques with noncritical stenosis in the left circumflex coronary artery, an ostial stenosis of $90 \%$ of the right coronary artery, but $90 \%$ stenosis at the proximal portion of the left anterior descending coronary artery (Figure 1).

After general anesthesia surgery was performed via a median sternotomy. The aorta, and superior and inferior venae cava were cannulated, and 


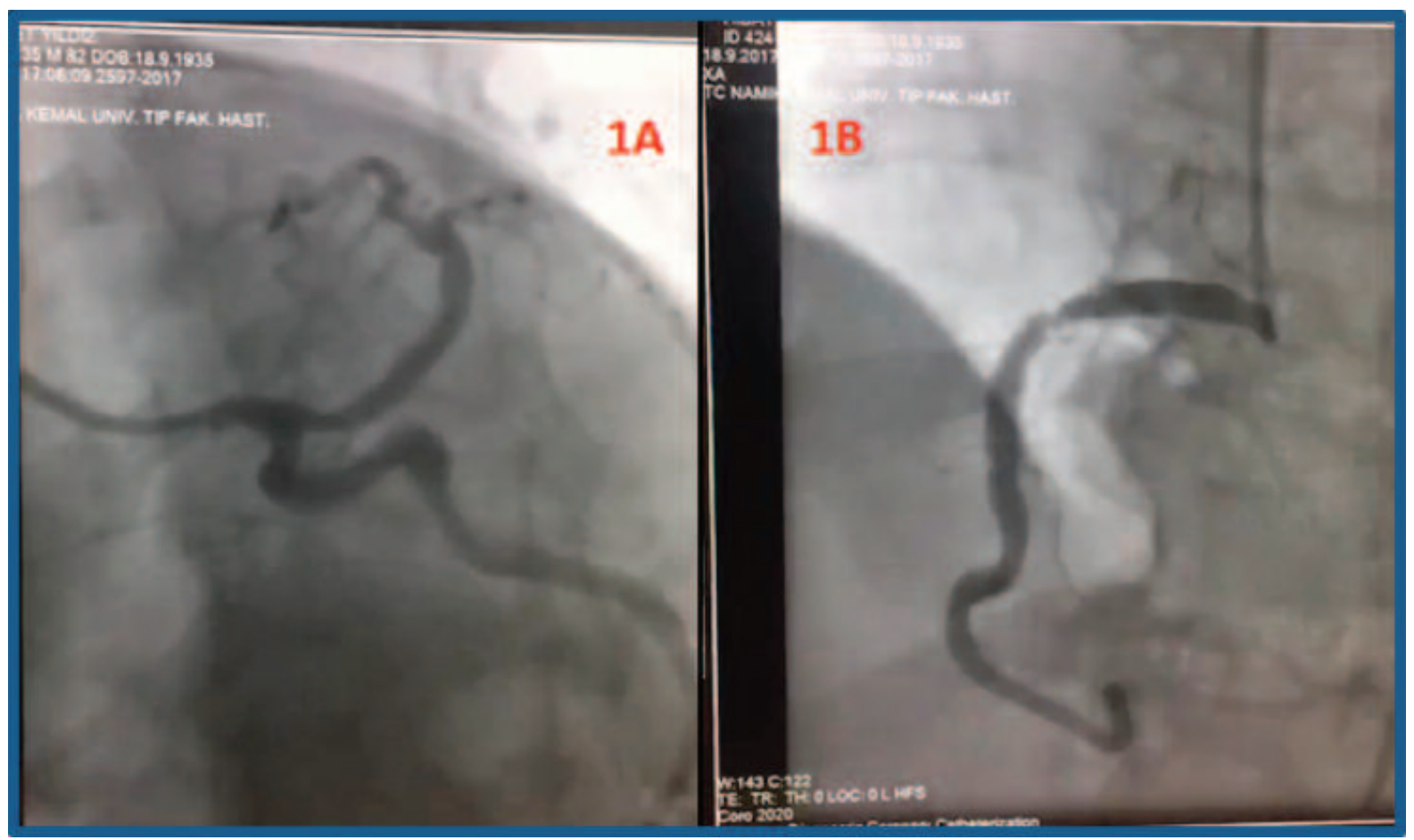

Figure 1. Coronary angiography shows demonstrating severe stenosis in the left anterior descending coronary artery (A) and the right coronary artery $(\mathrm{B})$.

cardiopulmonary bypass with moderate hypothermia $\left(28-30^{\circ} \mathrm{C}\right)$ was instituted. To minimize the risk of developing perioperative embolism the heart was gently handled during the operation. First the left atrial mass excised via interatrial groove approach (Figure 2 ). Excision of the tumor was performed with its pedicle originated from the left side of the atria. We performed coronary artery bypass grafting after the closure of the left atriotomy. For coronary revascularization of left descending coronary artery the internal thoracic artery was used and a saphenous vein graft anastomosed to aorto-right coronary position. The postoperative course was uneventful without any complication.

\section{Discussion}

We have presented a rare case of concomitant atherosclerotic coronary artery disease and left atrial myxoma. Moreover, this patient was the oldest patient

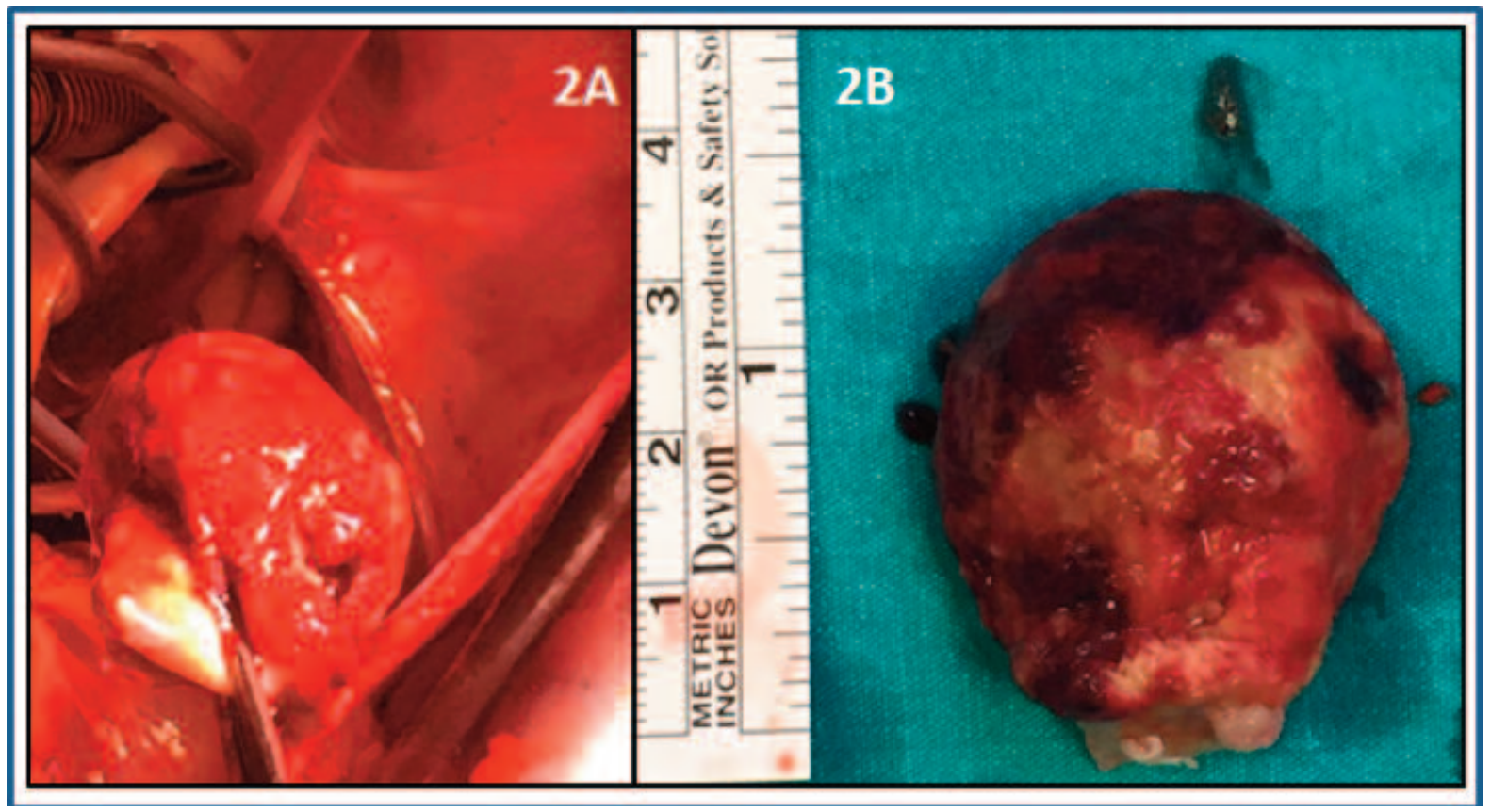

Figure 2. Appearance of the atrial mass during surgical excision (A). Macroscopic view of the surgically removed myxoma (B).). 
with coronary artery disease associated myxoma according to literature review. Atrial myxomas are the most common primary heart tumors $[2,3]$. However, the incidence of atrial myxomas ranges between 0.5 and 1 per million of population/year and mean age of presentation has been reported between 40 to 60 years [1].

Left atrial myxoma may or may not produce characteristic findings on physical examination. The myxoma symptoms depend on mitral valve obstruction or distal organ embolization [4]. Embolization is generally seen in central nervous system. But other organs such as the liver, spleen, kidney, retina, coronary vessels, and distal arterial tree can be affected [4]. Chest pain and dyspnea were the major symptoms of the patient. Clinical findings were thought to be the result of partial cardiac obstruction in addition to coronary artery disease. In case of acute coronary syndromes and left heart myxoma, coronary embolization should always be considered [5]. The incidence of coronary embolization of myxomas is $0.06 \%$. There was no evidence of major distal embolism in our patient. She had angina and shortness of breath at the same time. So we decided to operation because of clinical deterioration.

The prevalence of coronary atherosclerotic disease in patients with myxoma ranges between $20.3 \%$ and $36.6 \%$ [6-9]. In this report the patient had coronary artery disease according to coronary angiography 3 years ago. But myxoma not reported in previous echocardiography note.

\section{Conclusions}

Coronary angiography is required in elderly patients who will undergo other cardiac operations because of the risk of synchronous coronary artery disease. Similarly, echocardiography is also required in patients with coronary artery disease, because of the risk of synchronous cardiac lesion, as seen in our case.

\section{Informed consent}

Written informed consent was obtained from the patient for the publication of this case report.

\section{Conflict of interest}

The authors declared that there are no potential conflicts of interest with respect to the research, authorship, and/or publication of this article.

\section{References}

[1] Bhan A, Mehrotra R, Choudhary SK, Sharma R, Prabhakar D, Airan B, et al. Surgical experience with intracardiac myxomas: long-term follow-up. Ann Thorac Surg 1998;66:810-3.

[2] Moyssakis I, Anastasiadis G, Papadopoulos D, Margos P, Votteas V. Second recurrence of cardiac myxoma in a young patien: a case report. Ann Thorac Surg 2005;101:501-2.

[3] Toktaş F, Yavuz Ş, Özsin KK, Sanrı US, Türk T, Göncü MT. Cardiac myxomas: an analysis of 39 patients. Eur Res J 2017;3:227-33.

[4] Nurözler F, Tandoğan A, Yamak B. [Huge left atrial myxoma with minimal symptoms: case report]. Turk Gogus Kalp Dama 2003;11:523. [Article in Turkish]

[5] Fang BR, Chang CP, Cheng CW, Yang NI, Shieh MC, Lee N. Total detachment of cardiac myxoma causing saddle embolization and mimicking aortic dissection. Jpn Heart J 2004;45:359-63.

[6] Kocaturk H, Karaman A, Bayram E, Çolak MC, Yurtman V. Left atrial myxoma and concomitant atherosclerotic coronary artery disease. Eurasian J Med 2009;41:202-4.

[7] Erdil N, Ates S, Cetin L, Demirkilic U, Sener E, Tatar H. Frequency of left atrial myxoma with concomitant coronary artery disease. Surg Today 2003;33:328-31.

[8] Yavuz S, Toktas F, Eris C, Ata Y, Turk T, Goncu T. Left atrial mass in a patient with previous coronary artery bypass grafting. Journal-CVS 2014;2:7-9.

[9] Gismondi RA, Kaufman R, Correa GA, Nascimento C, Weitzel LH, Reis JO, et al. [Left atrial myxoma associated with obstructive coronary artery disease]. Arq Bras Cardiol 2007;88:e1-3. [Article in Portuguese] 